\title{
Improvements in IVF in women of advanced age
}

\author{
Norbert Gleicher 1,2,3, Vitaly A Kushnir1,4, David F Albertini1,5 and David H Barad1,2 \\ 1The Center for Human Reproduction, New York, New York, USA \\ ${ }^{2}$ The Foundation for Reproductive Medicine, New York, New York, USA \\ ${ }^{3}$ The Brivanlou Stem Cell Biology and Molecular Embryology Laboratory, The Rockefeller University, \\ New York, New York, USA \\ ${ }^{4}$ Department of Obstetrics and Gynecology, Wake Forest University, Winston Salem, North Carolina, USA \\ ${ }^{5}$ Department of Molecular and Integrative Physiology, The University of Kansas Medical Center, Kansas \\ City, Kansas, USA
}

Correspondence

should be addressed to

N Gleicher

Email

ngleicher@rockefeller.edu

\begin{abstract}
Women above age 40 years in the US now represent the most rapidly growing age group having children. Patients undergoing in vitro fertilization (IVF) are rapidly aging in parallel. Especially where egg donations are legal, donation cycles, therefore, multiply more rapidly than autologous IVF cycles. The donor oocytes, however, are hardly ever a preferred patient choice. Since with use of own eggs, live birth rates decline with advancing age but remain stable (and higher) with donor eggs, older patients always face the difficult and very personal choice between poorer chances with own and better chances with donor oocytes. Physician contribution to this decision should in our opinion be restricted to accurate outcome information for both options. Achievable pregnancy and live birth rates in older women are, however, frequently underestimated, thereby mistakenly biasing fertility providers, private insurance companies and even regulatory government agencies. Restriction on access to IVF for older women is then often the consequence. In this review, we summarize the limited published data on best treatments of 'older' ovaries, while also addressing treatment approaches that should be avoided in older women. This focused review, therefore, to a degree is subjective. Research addressing aging ovaries in IVF has been disappointingly sparse, and has in our opinion too heavily concentrated on methods of embryo selection (ES), which, especially in older women, not only fail to improve IVF outcomes, but actually, negatively affect live birth chances. We conclude that, aside from breakthroughs in gamete creation, only pharmacological interventions into early (small growing follicle stages) follicle maturation will offer new potential to positively impact oocyte and embryo quality and, therefore, IVF outcomes. Research, therefore, should be accordingly redirected.
\end{abstract}

\section{Key Words}

- advanced female age

- in vitro fertilization

- live birth rate

- clinical pregnancy rate

- premature ovarian aging

- embryo selection

\section{Background}

\section{Female age}

Women above age 40 years over the last 10 years has represented in the US the most rapidly growing age category having children. It, therefore, is not surprising that patient populations in fertility centers, including women undergoing in vitro fertilization (IVF), have been aging in parallel. Rapidly increasing ages among IVF patients

Published by Bioscientifica Ltd
Journal of Endocrinology (2016) 230, F1-F6 
are further driven by the fact that young and uncomplicated IVF patients now conceive rapidly, while older and more difficult patients, due to lower pregnancy chances, require more cycle attempts and, therefore, proportionally accumulate (Gleicher et al. 2014). IVF populations in developed countries are, therefore, aging rapidly.

Declines in oocyte yields and oocyte quality (i.e. 'ovarian aging') are the primary reasons for deteriorating IVF outcomes with advancing female age, with oocyte quality determining most of embryo quality and embryo quality determining most of pregnancy and live birth chances. In IVF, female age, therefore, is a principal outcome-criterion for clinical providers, insurance companies, and even regulatory government agencies. Unfortunately, it is also widely used to restrict access to autologous IVF, often prematurely pressuring patients to consider younger donor eggs as the only option to conceive. Proponents of such an approach argue that, because of low pregnancy and live birth chances at advanced female age, responsible resource allocation does not support utilization of autologous IVF beyond certain age thresholds (reviewed in Gleicher et al. 2014).

The 2013 US Society for Assisted Reproductive Technology (SART) data, the last available full annual data set, indicated that only $6.6 \%$ of fresh nondonor IVF cycles were performed in women above age 42 years. The recently published, still preliminary and incomplete 2014 data set suggests that this number increased to $8.4 \%$.

Small percentages of autologous IVF cycles in women above age 42 years, contrasted by rapidly multiplying donor-recipient cycles (SART website https://www.sartcorsonline.com/rptCSR_PublicMultYear. aspx?ClinicPKID=0), reflect significant ongoing resistance to treatment of older women at most US IVF centers. For research purposes,we have recently been given access to annual SART data for the years 2004-2014.
These data demonstrated that $10.2 \%$ of all IVF cycles in women up to age 42 utilized donor oocytes, though 49.7\% in women above age 42 years (unpublished data).

What causes this skepticism about the use of autologous oocytes above age 42 years is unclear, but older patients frequently report to have been advised by their physicians that live birth chances with IVF are equal to those of insemination cycles, that is, ranging only in the 1-2\% (The American Society for Reproductive Medicine, ASRM, defines futility as live birth chances below 1.0\%). Achievable reported IVF outcomes are, however, clearly better than that, and even higher than reported in above referenced national SART data (Table 1).

As low live birth chances are considered uneconomical, women above age 42 are frequently denied IVF access, in the US by coverage criteria issued by insurance companies, in many European countries, and at least one large Canadian province by legislative mandates or government agency agreements with the medical IVF community, offering government insurance coverage for IVF in return for age restrictions for access. Some Scandinavian countries, indeed, already after ages of 40-41 restrict women's access to IVF. To the best of our knowledge, no such access restrictions to fertility treatments exist anywhere in the world based on male age (Gleicher et al. 2014).

Accurate outcome statistics for women with 'older ovaries', therefore, appear to be of considerable importance to allow physicians, insurance carriers, as well as government agencies to reach appropriate policy decisions as to what they consider best and cost-effective infertility care in older women.

In doing so, age is, however, not the only important outcome predictor of IVF. The so-called functional ovarian reserve (FOR), representing the small growing follicle pool up to small preantral follicle stages, is almost as important. If FOR is diminished for age, a patient

Table 1 Preliminary US national 2014 IVF outcomes per age category, as reported by SART*.

\begin{tabular}{|c|c|c|c|c|c|}
\hline & \multicolumn{5}{|c|}{ Patient age (years) } \\
\hline & $<35$ & $36-37$ & $38-40$ & $41-42$ & $>42$ \\
\hline Cycle starts $(n)$ & 40,805 & 21,137 & 20,274 & 10,870 & 8514 \\
\hline Live births (\%) & 42.6 & 33.9 & 22.3 & 12.0 & 3.6 \\
\hline$(\mathrm{Cl})$ & $(42.1-43.1)$ & $(33.3-34.5)$ & $(21.7-22.9)$ & $(11.4-12.6)$ & $(3.8-4.4)$ \\
\hline
\end{tabular}

*These data are considered preliminary by SART since $1.4 \%$ of cycle outcomes are reported as 'delayed outcomes'. They reflect 'intent-to-treat' analysis, which means that outcomes are reported with reference cycle start. This is an important point to consider when assessing IVF outcomes in older women because the older a patient, the more likely will her cycle be cancelled before embryo transfer, either because her ovaries do not respond to stimulation or no transferrable embryos are obtained. Women between age 42 and 49 have an approximately one-third chance of cycle cancellation. Those patients who do produce embryos for transfer, especially if producing more than three, will therefore, have better live birth outcomes than reported in this table (Gleicher et al. 2015a). It is also important to note that here reported outcomes by age categories are all inclusive. Within each age category, there are women with normal age-specific FOR and low FOR. Women with low FOR will, of course, do less well than women with normal FOR.

http://joe.endocrinology-journals.org DOI: 10.1530/JOE-16-0105 (c) 2016 Society for Endocrinology Printed in Great Britain
Published by Bioscientifica Ltd 
is considered to suffer from premature ovarian aging (POA) or occult primary ovarian insufficiency (OPOI), found in approximately $10 \%$ of all women independent of race and ethnic background. A clinical diagnosis is usually reached by demonstrating abnormally high agespecific follicle-stimulating (FSH) and/or abnormally low anti-Müllerian hormone (AMH) (Gleicher et al. 2011). Interestingly, POA/oPOI and older women demonstrate very similar intrafollicular molecular profiles of premature luteinization (Wu Y-G, Barad DH, Kushnir VA, Wang Q, Zhang L, Darmon S, Albertini DF \& Gleicher N, unpublished observations), further discussed below.

Unfortunately, national IVF outcome reports do not yet accurately reflect FOR. Outcome reports by age categories, therefore, are all inclusive, and include patients with normal as well as abnormal FOR (Table 1). Within any given age category, women with low FOR will, however, always do worse than women with normal FOR.

\section{Recent developments}

\section{What is possible?}

The literature does not define achievable clinical pregnancy and live birth rates in poor prognosis patients well, since most IVF centers do not treat women at very advanced ages and/or with very low FOR. Published outcome data in such patient populations, therefore, come mostly from our center which, based on the US national registry data, proportionally serves the oldest patient population in the nation.

Using this patient population, in 2011 we reported on 128 consecutive patients with extremely low FOR (mean FSH $15.7 \pm 11.1 \mathrm{mIU} / \mathrm{mL}$; mean AMH $0.2 \pm 0.1 \mathrm{ng} / \mathrm{mL}$ ) at mean the age $40.8 \pm 4.1$ years. Though at that point the worst prognosis patient population ever reported in the literature, these women still achieved clinical pregnancies in $7.9 \%$ of cycle starts (95\% CI: 4.9-11.9) and a cumulative rate in three cycles of $15.6 \%$ (95\% CI: 9.8-23.1) (Weghofer et al. 2011). Notably, age 43 demarked a clear cutoff between better and poorer pregnancy chances, with women under that age achieving pregnancies in 14.3\%, and those between 43 and older in only $3.4 \%$. Clinical pregnancy $(P=0.013)$ and live birth rates $(P=0.036)$ were, thus, significantly lower in women above age 43 years.

While these were quite poor IVF outcomes, even above age 43 years they still exceeded previously noted widely quoted 1-2\% live birth rate as well as ASRM's definition of futility in IVF, which is a live birth chance of less than $1 \%$. A recent study, however, further clarified the picture, offering directions as to which older women may have higher than only average chances. We reported on 483 very poor prognosis patients who in 381 fresh IVF cycles produced at least one cleavage stage embryo for transfer. This analysis, thus, excluded approximately one-third of older women who had started IVF cycles, but were cancelled before embryo transfer because not even one embryo was produced in the cycle. Once again, this publication represented at that date the most adversely selected patient population reported in the literature (Gleicher et al. 2015a). However, it is important to note that in an attempt to identify patients with above average chances for live births, this outcome analysis was done with reference point embryo transfer rather than cycle start (intent to treat).

Up to age 42 , even availability of only one single embryo for transfer offered excellent to reasonably good live birth chances of $33.3 \%$ under age 35 years and $6.4 \%$ at $41-42$ years. Above age 43 , women, however, required at least three embryos to achieve a live birth rate of $7.4 \%$. Those with three or more embryos, however, did relatively well up to almost age 47, for the first time demonstrating the importance of available embryos numbers for transfer above age 43 .

The study, however, also reconfirmed that age 43 represented a sudden break point for IVF outcomes in older women. The very obvious next question was why?

\section{The changing intrafollicular molecular profile with age}

A recently published study in this journal answered this question at least in part, investigating the molecular follicle environment in IVF cycles of women at different ages, and demonstrating that follicular maturation processes in older women accelerate. Gene expression studies by real-time PCR and western blot in follicular fluid granulosa cells (GCs), related to gonadotropin activity, steroidogenesis, apoptosis, and luteinization, demonstrated in women above age 43 years convincing evidence of prematurely luteinization at already relative small follicle sizes. At in vitro culture, GCs from older women exhibited lower proliferation and increased apoptosis, while FSH supplementation stimulated cell growth and prevented luteinization (Wu et al. 2015).

Routine ovulation induction with human chorionic gonadotropin (hCG) at lead follicle sizes of $19-22 \mathrm{~mm}$ in older women, thus, resulted in prematurely luteinized follicles which, in turn, produced 'overmature', poor quality oocytes. Giving the hCG trigger earlier at approximate lead follicle sizes of $16 \mathrm{~mm}$, significantly reduced molecular

Published by Bioscientifica Ltd 
evidence of premature luteinization, and approximately doubled clinical pregnancy rates per transfer in women above age 43 years from 7.7 to $15.5 \%$ (Wu et al. 2015).

Two follow-up studies confirmed and expanded upon these results. In the first study, we reaffirmed above age 43 that $16-18 \mathrm{~mm}$ was, indeed, best lead follicle size to trigger ovulation with hCG. Both, smaller $(<16 \mathrm{~mm})$ and larger follicle sizes $(>18.5 \mathrm{~mm})$, produced significantly lower clinical pregnancy rates. This finding was of particular interest because molecular evidence of premature luteinization continued to decrease quantitatively with ovulation triggers at even smaller follicle sizes below $16 \mathrm{~mm}$; yet, pregnancy rates were similarly poor to those of women with standard follicle size retrievals $(>18.5 \mathrm{~mm})$ and with obvious molecular evidence of premature luteinization (Wu Y-G, Barad DH, Kushnir VA, Wang Q, Zhang L, Darmon S, Albertini DF \& Gleicher N, unpublished observations). Poor pregnancy results in IVF can, therefore, also have other potential causes. If follicles are triggered too early, one such cause may be insufficient cytoplasmic maturation of oocytes (Wu et al. 2007).

In a second follow-up study, young women with $\mathrm{POA} / \mathrm{OPOI}$ were found to demonstrate the same gene and protein expression signature of premature luteinization, previously observed in older women over age 43. Early oocyte retrieval at $16-18 \mathrm{~mm}$ lead follicle size, however, not only doubled, but tripled pregnancy rates in comparison to controls. Like in older women, early retrievals with $16 \mathrm{~mm}$ hCG trigger significantly reduced molecular evidence of premature luteinization (Wu Y-G, Barad DH, Kushnir VA, Wang Q, Zhang L, Darmon S, Albertini DF \& Gleicher N, unpublished observations). In the truest sense of the word, POA/OPOI women, thus, really prematurely age their follicles/ovaries.

Whatever the underlying cause, women with low FOR at our center, therefore, are now triggered almost routinely at $16-18 \mathrm{~mm}$ lead follicle sizes.

\section{Controversies}

Quite a number of improvements have been proposed for IVF over the last decade. Some have remained controversial. We here will address those we consider most important.

\section{Androgen supplementation of women with low FOR}

Casson et al. (2000) were the first to suggest that androgen supplementation with DHEA may beneficially affect low FOR and, therefore, IVF outcomes in older women.
Since then, low FOR has been defined as a relatively hypoandrogenic condition in need of androgen supplementation (Gleicher et al. 2013), and various animal models established the importance of appropriate testosterone levels for normal growth and maturation of small growing follicles (recently reviewed in this journal by Prizant et al. 2014). DHEA effects have been attributed to its conversion to testosterone, which synergistically enhances FSH effects on GCs through the androgen receptor (reviewed in Prizant et al. 2014, Shohat-Tal et al. 2015).

Absence of properly powered prospectively randomized studies of androgen supplementation in women with low FOR is the main reason why this treatment approach has remained controversial. Though a number of randomized studies have been published, none achieved adequate power in number of participating patients and/or involved appropriately selected patient populations. For that reason, meta-analyses either had to conclude that not enough data existed to reach conclusions or that no treatment effects were apparent. Small as well as large animal studies, however, repeatedly demonstrated highly significant effects (animal as well as human studies are in detail reviewed in Prizant et al. 2014).

Our center's above-noted IVF outcomes in very poor prognosis patients would, however, have been impossible without appropriate DHEA (or testosterone) supplementation (please note the authors' listed competing interests in reference to this statement).

\section{The concept of embryo selection}

It is reasonable to assume that best embryos offer highest pregnancy and live birth chances. Embryo selection (ES) has, therefore, for decades been a guiding principle in IVF, initially primarily based on routine morphological assessments of cleavage stage (day 3) embryos. Because it promised improved implantation rates and, therefore, less need for multiple-embryo transfer, the next ES method to achieve wide popularity was prolonged embryo culture to blastocyst stage (BEC) (Gardner et al. 1998).

Subsequent BEC studies in general infertility populations, however, were unable to confirm originally reported outcome benefits in good prognosis patients, and it is now widely accepted that BEC marginally improves immediate pregnancy and live birth rates in IVF, though only in good prognosis patients (young women with good quality day 3 embryos in large numbers). Transfer of cleavagestage (day 3) embryos, however, in comparison to BEC in all patient populations (including good prognosis patients), demonstrates superior cumulative pregnancy rates from

Published by Bioscientifica Ltd. 
a complete embryo cohort obtained in a single IVF cycle (reviewed in Gleicher et al. 2015b). This observation suggests that some embryos, which do not survive prolonged culture to blastocyst stage can probably still produce normal pregnancies and deliveries if transferred on day 3.

Many IVF laboratories, nevertheless, have adopted BEC as their principal embryo culture method and indiscriminately apply it to all patients. Because poor prognosis patients usually produce small embryo yields, they are disproportionally and more severely negatively affected by BEC than average and good prognosis patients. BEC, therefore, actually reduces immediate IVF pregnancy chances in older women and other poor prognosis patients.

With claims to further improve ES in association with BEC, additional ES methods have been added to BEC, including preimplantation genetic screening (PGS), closed incubation systems with time-lapse imaging, and others. In older women and other poor prognosis patients, these additional ES methods only further augment the potential shortcomings of BEC, and further negatively affect IVF outcomes (Gleicher et al. 2015b).

\section{Future directions}

Due to inappropriate preselection of study populations, incorrect outcome reporting in association with IVF has accelerated in recent years (Gleicher et al. 2016). Since patient preselection via BEC, PGS, and other ES methods disproportionally excludes older women and other poor prognosis patients from consideration (they often do not reach embryo transfer), outcome reports in the literature (with reference point embryo transfer) claiming outcome benefits for these new additions to IVF are often misleading and highly exaggerated.

Such misleading reports are particularly hurtful to older patients and other poor prognosis patients because they usually produce only small oocye/embryo yields. Avoidable embryo losses, whether by transferring at cleavage rather than blastocyst stage or avoiding falsepositive results by omitting PGS, is particularly important in women with very small oocyte/embryo numbers. These ES methods, therefore, especially in older women, should be avoided.

ES methods also have to be questioned on biological grounds: a principal reason is the length of follicle maturation after follicle recruitment (Fig. 1). To assume that ES can still significantly affect IVF outcomes after months of preceding follicle/oocyte maturation, appears biologically implausible. It appears more reasonable to assume that, to be clinically effective, therapeutic interventions into follicle maturation have to take place at much earlier developmental stages, like small growing follicle stages.

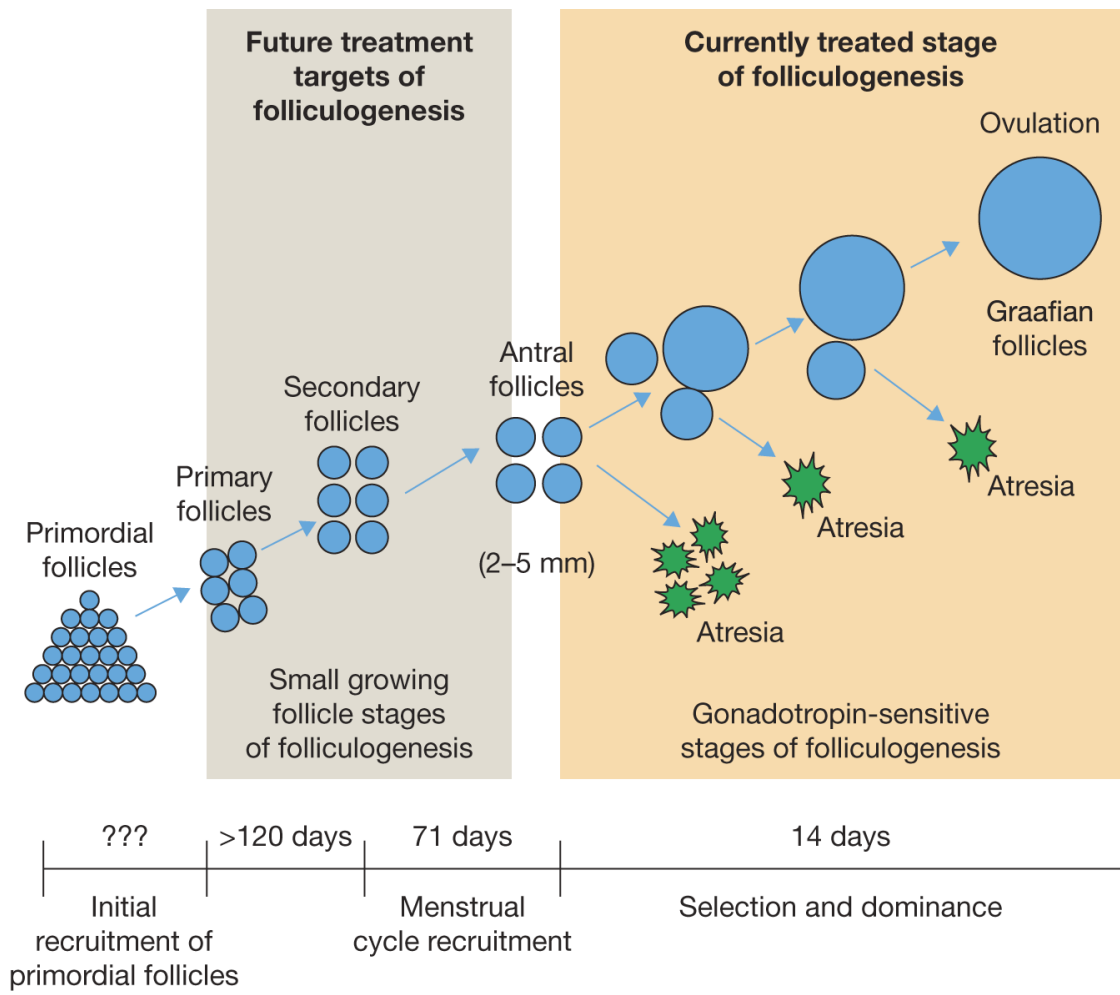

\section{Figure 1}

Current and future therapeutic targets of interventions into folliculogenesis. The figure demonstrates the approximate time span (in days) for the different stages of follicle maturation. Modern infertility treatments of the last 50 years almost exclusively only intervened pharmacologically at the gonadotropin-sensitive last 14 days of follicle maturation. In pointing out future directions for fertility treatments of older women, we here argue that effective therapeutic interventions have to be directed at much earlier stages of follicle maturation, involving small growing follicle stages. http://joe.endocrinology-journals.org DOI: 10.1530/JOE-16-0105
C 2016 Society for Endocrinology Printed in Great Britain 
To further improve IVF cycle outcomes in older women, clinicians have to become more willing to provide services to older women, and to learn what does and does not works. Had such willingness not existed during early stages of IVF in the 1980s, we would still not treat women over age 38 because that age limit was initially considered unbreachable.

Though limited and largely restricted to our center, here presented IVF outcome data in older women should, hopefully, be convincing enough to encourage a more active pursuit of such treatments. We, however, also believe that significant further improvements in IVF outcomes will come about only with radically new therapeutic approaches that go beyond the field's exclusive concentration over the last 60 years on the gondadotropindependent last 2 weeks of follicle maturation. It appears time to carry therapeutic interventions into much earlier stages of follicle recruitment and follicle maturation. The Pharma industry's lack of interest in doing so over the last two decades, indeed, appears somewhat surprising, and is probably at least partially responsible for worldwide stagnation in IVF outcomes over the last decade.

The experience described briefly above with the androgen supplementation of small growing follicles is probably currently the best example for such early interventions into follicle maturation. If IVF outcomes are to be further improved, more and better quality oocytes will have to be matured in ovaries of older women and other poor prognosis patients. Short of achieving breakthrough in in vitro gamete production, this will only be achieved by intervening earlier into the follicle maturation process than current practice calls for.

\section{Declaration of interest}

N G and D H B are co-inventors on a number of pending and already awarded US patents claiming therapeutic benefits from androgen supplementation in women with low functional ovarian reserve (LFOR) and relating to the $F M R 1$ gene in a diagnostic function in female fertility. Both receive royalties from Fertility Nutraceuticals, LLC, in which N G also holds shares. N G, D H B, and V A K are also co-inventors on three pending $\mathrm{AMH}$-related patent application. All authors received in the past research support, travel funds, and speaker honoraria from various Pharma and/or medical device companies, none though related to here discussed subjects. They report no other potential conflicts with here reported manuscript.

\section{Funding}

This manuscript received no outside funding beyond salary support for the authors from The Center for Human Reproduction.

\section{Author contribution statement}

All authors contributed to the concept of the manuscript. N G wrote the manuscript with significant contributions by all other authors. All authors approved of the final manuscript.

\section{References}

Casson PR, Lindsay MS, Pisarska MD, Carson SA \& Buster JE 2000 Dehydroepiandrosterone supplementation augments ovarian stimulation in poor responders: a case series. Human Reproduction $\mathbf{1 5}$ 2129-2132. (doi:10.1093/humrep/15.10.2129)

Gardner DK, Vella P, Lane M, Wagley L, Schlenker T \& Schoolcraft WB 1998 Culture and transfer of human blastocysts increases implantation rates and reduces the need for multiple embryo transfers. Fertility and Sterility 69 84-88. (doi:10.1016/S00150282(97)00438-X)

Gleicher N, Weghofer A \& Barad DH 2011 Defining ovarian reserve to better understand ovarian aging. Reproductive Biology and Endocrinology 9 23. (doi:10.1186/1477-7827-9-23)

Gleicher N, Kim A, Weghofer A, Kushnir VA, Shohat-Tal A, Lazzaroni E, Lee HJ \& Barad DH 2013 Hypoandrogenism is associated with diminished functional ovarian reserve. Human Reproduction $\mathbf{2 8}$ 1084-1091. (doi:10.1093/humrep/det033)

Gleicher N, Kushnir VA, Weghofer A \& Barad DH 2014 The "graying" of infertility services: an impending revolution nobody is ready for Reproductive Biology and Endocrinology 12 63. (doi:10.1186/14777827-12-63)

Gleicher N, Vega MV, Darmon SK, Weghofer A, Wu YG, Wang Q, Zhang L, Albertini DF, Barad DH \& Kushnir VA 2015a Livebirth rates in very poor prognosis patients, who are defined as poor responders under Bologna criteria, with nonelective single embryo, two-embryo and three or more embryos transferred. Fertility and Sterility 104 1435-1441. (doi:10.1016/j. fertnstert.2015.08/023)

Gleicher N, Kushnir VA \& Barad DH $2015 b$ Is it time for a paradigm shift in understanding embryo selection? Reproductive Biology and Endocrinology 13 3. (doi:10.1186/1477-7827-13-3)

Gleicher N, Kushnir VA \& Barad DH 2016 The impact of patient preselection on reported IVF outcomes. Journal of Assisted Reproduction and Genetics 33 455-459. (doi:10.1007/s10815-0160673-9)

Prizant H, Gleicher N \& Sen A 2014 Androgen action in the ovary: balance is key. Journal of Endocrinology 222 R141-R151. (doi:10.1530/ JOE-14-0296)

Shohat-Tal A, Sen A, Barad DH, Kushnir VA \& Gleicher N 2015 Genetics of androgen metabolism in women with infertility and hypoandrogenism. Nature Reviews Endocrinology $11429-441$. (doi:10.1038/nrendo.2015.64)

Weghofer A, Dietrich W, Barad DH \& Gleicher N 2011 Live birth chances in women with extremely low-serum anti-Mullerian hormone levels. Human Reproduction 26 1905-1909. (doi:10.1093/ humrep/der134)

Wu Y-G, Liu Y, Zhou P, Lan GC, Han D, Miao DQ \& Tan JH 2007 Selection of oocytes for in vitro maturation by brilliant cresyl blue staining: a study using the mouse model. Cell Research 17 722-731. (doi:10.1038/cr.2007.66)

Wu Y-G, Barad DH, Kushnir VA, Lazzaroni E, Wang Q, Albertini DF \& Gleicher N 2015 Aging-related premature luteinization of granulosa cells is avoided by early oocyte retrieval. Journal of Endocrinology $\mathbf{2 2 6}$ 167-180. (doi:10.1530/JOE-15-0246)

Received in final form 22 April 2016

Accepted 6 May 2016

Accepted Preprint published online 6 May 2016 http://joe.endocrinology-journals.org

DOI: $10.1530 / \mathrm{JOE}-16-0105$
C 2016 Society for Endocrinology Printed in Great Britain 